Check for updates

Cite this: RSC Adv., 2018, 8, 26218

Received 9th May 2018

Accepted 11th July 2018

DOI: $10.1039 / \mathrm{c} 8 \mathrm{ra03972k}$

rsc.li/rsc-advances

\section{Hydroxyapatite nanowire/collagen elastic porous nanocomposite and its enhanced performance in bone defect repair $\dagger$}

\begin{abstract}
Tuan-Wei Sun, Ying-Jie Zhu (D)* and Feng Chen*
The synthetic bone grafts that mimic the composition and structure of human natural bone exhibit great potential for application in bone defect repair. In this study, a biomimetic porous nanocomposite consisting of ultralong hydroxyapatite nanowires (UHANWs) and collagen (Col) with 66.7 wt\% UHANWs has been prepared by the freeze drying process and subsequent chemical crosslinking. Compared with the pure collagen as a control sample, the biomimetic UHANWs/Col porous nanocomposite exhibits significantly improved mechanical properties. More significantly, the rehydrated UHANWs/Col nanocomposite exhibits an excellent elastic behavior. Moreover, the biomimetic UHANWs/Col porous nanocomposite has a good degradable performance with a sustained release of $\mathrm{Ca}$ and $\mathrm{P}$ elements, and can promote the adhesion and spreading of mesenchymal stem cells. The in vivo evaluation reveals that the biomimetic UHANWs/Col porous nanocomposite can significantly enhance bone regeneration compared with the pure collagen sample. After 12 weeks implantation, the woven bone and lamellar bone are formed throughout the entire UHANWs/Col porous nanocomposite, and connect directly with the host bone to construct a relatively normal bone marrow cavity, leading to successful osteointegration and bone reconstruction. The as-prepared biomimetic UHANWs/Col porous nanocomposite is promising for applications in various fields such as bone defect repair.
\end{abstract}

\section{Introduction}

Millions of people worldwide need bone grafts to reconstruct large bone defects caused by trauma, tumor surgery and infection each year. ${ }^{1-4}$ Nowadays, autologous bone grafting and allogeneic bone grafting remain the most preferable treatments for large bone defects. However, the clinical applications of the autograft and allograft are limited by the donor-site morbidity, limited supply, risks of infection and immunological reaction..$^{5-7}$ To overcome the shortcomings of the autograft and allograft, various types of synthetic bone graft substitutes have been extensively investigated to fulfil bone integration and bone regeneration in vivo. ${ }^{8-10}$

To achieve successful bone regeneration, the synthetic bone graft substitutes should be osteoconductive and osteoinductive, that is, the ideal synthetic bone graft substitutes should recruit progenitor cells and facilitate their proliferation and osteogenic differentiation, support the formation of new blood vessels and bone tissue, and be gradually metabolized and replaced by the

State Key Laboratory of High Performance Ceramics and Superfine Microstructure, Shanghai Institute of Ceramics, Chinese Academy of Sciences, Shanghai 200050, P. R. China. E-mail: y.j.zhu@mail.sic.ac.cn; fchen@mail.sic.ac.cn; Fax: +86-2152413122; Tel: +86-21-52412616

$\dagger$ Electronic supplementary information (ESI) available. See DOI: $10.1039 / \mathrm{c} 8 \mathrm{ra} 03972 \mathrm{k}$ newly formed tissues. ${ }^{11-13}$ In addition, the ideal synthetic bone graft substitute should possess excellent mechanical properties to allow surgical handling and fixation. ${ }^{\mathbf{1 1} \mathbf{1 4}}$ To achieve these goals, it is important to develop bone graft substitutes mimicking the chemical composition and structure of human natural bone. The natural bone is an inorganic-organic biocomposite with $\sim 70 \mathrm{wt} \%$ minerals mainly hydroxyapatite $\left(\mathrm{HAP}, \mathrm{Ca}_{10}\left(\mathrm{PO}_{4}\right)_{6}(\mathrm{OH})_{2}\right)$ and $\sim 30 \mathrm{wt} \%$ organic matrix (mainly type I collagen). More interestingly, the natural bone is hierarchically constructed by HAP nanocrystals and collagen matrix at the macro-, micro-, and nanoscales, which endows the natural bone with excellent mechanical properties. ${ }^{3,9,15}$ Thus, it is a rational strategy to develop bone graft substitutes based on biomimetics.

The synthetic HAP is similar to the major inorganic component of the natural bone in terms of chemical composition and structure, and has high biocompatibility, excellent cell adhesion, and high osteoconductivity. ${ }^{\mathbf{1 6 - 1 8}}$ Moreover, collagen, as the organic constituent of the bone extracellular matrix, is a promising biomaterial owing to its excellent biocompatibility, cell affinity and biodegradability. ${ }^{19-21}$ Consequently, the HAP/ collagen (HAP/Col) composite scaffolds can chemically and structurally mimic the natural bone, and have excellent bioactivity, biodegradability and osteoinductive activity. ${ }^{11,13,22}$ So far, the biomimetic HAP/Col composite scaffolds with high porosity are typically fabricated via lyophilisation, which is beneficial for cell infiltration, neovascularization and new bone ingrowth. ${ }^{23-25}$ 
However, the lyophilized HAP/Col scaffolds usually exhibit small pore sizes and poor mechanical properties. ${ }^{11,22,26}$

Recent reports indicate that the addition of one-dimensional HAP nanostructured materials such as HAP whiskers and nanowires can significantly improve the mechanical properties and biological responses of the composite biomaterials. ${ }^{11,18,22,27}$ More significantly, the highly flexible ultralong HAP nanowires (UHANWs) with lengths of several hundred micrometres were prepared by the calcium oleate precursor solvothermal method by this research group,,$^{28-30}$ and exhibit a great potential in constructing different types of biomaterials with enhanced mechanical properties and improved biological responses. ${ }^{18,27,31}$ In addition, the morphology of the UHANWs is similar to that of mineralized collagen fibrils in the natural bone. Thus, the UHANWs offer a promising prospect for the construction of biomimetic HAP/Col porous nanocomposite with enhanced mechanical properties and biological responses.

In our previous work, the UHANWs/collagen (UHANWs/Col) composite porous scaffolds with UHANWs weight fractions ranging from 0 to $100 \mathrm{wt} \%$ were synthesized, and exhibited significantly enhanced mechanical properties compared with the HAP nanorods/collagen scaffolds. ${ }^{32}$ In this study, inspired by the chemical composition and structure of the natural bone, a biomimetic UHANWs/collagen (UHANWs/Col) elastic porous nanocomposite with $66.7 \mathrm{wt} \%$ UHANWs has been prepared by the freeze drying and subsequent chemical crosslinking. Furthermore, the mechanical properties and cell attachment performance of the UHANWs/Col porous nanocomposite are investigated in detail. More importantly, the in vivo studies have been performed to evaluate the performance of the biomimetic UHANWs/Col porous nanocomposite in bone regeneration compared with the pure collagen sample. Compared with the pure collagen control sample, the biomimetic UHANWs/Col elastic porous nanocomposite exhibits significantly improved mechanical properties. More significantly, the rehydrated UHANWs/Col porous nanocomposite exhibits excellent elastic behavior. Moreover, the biomimetic UHANWs/Col porous nanocomposite has a good degradable performance with a sustained release of $\mathrm{Ca}$ and $\mathrm{P}$ elements, and can promote the adhesion and spreading of mesenchymal stem cells. The in vivo evaluation reveals that the biomimetic UHANWs/Col porous nanocomposite can significantly enhance bone regeneration compared with the pure collagen sample. After 12 weeks implantation, the woven bone and lamellar bone are formed throughout the entire UHANWs/Col porous nanocomposite, and the newly formed bone can connect directly with the host bone to construct a relatively normal bone marrow cavity, leading to successful osteointegration and bone reconstruction.

\section{Experimental section}

\subsection{Chemicals}

$\mathrm{CaCl}_{2}, \mathrm{NaOH}, \mathrm{NaH}_{2} \mathrm{PO}_{4} \cdot 2 \mathrm{H}_{2} \mathrm{O}$, and methanol were purchased from Sinopharm Chemical Reagent Co. Ltd. Absolute ethanol was obtained from Lingfeng Chemical Reagent Co. Ltd. Oleic acid was purchased from Aladdin Industrial Corporation. $\mathrm{N}$-(3dimethylaminopropyl)- $N$-ethylcarbodiimide hydrochloride
(EDC) and $N$-hydroxysuccinimide (NHS) were purchased from Sigma-Aldrich. The lyophilized collagen sponge was purchased from Chengdu Kele Biological Technology Co. All chemicals were used as received without further purification. Deionized water was used in all experiments.

\subsection{Synthesis of ultralong hydroxyapatite nanowires (UHANWs)}

UHANWs were synthesized by the calcium oleate precursor solvothermal method. ${ }^{28-30}$ In a typical experiment, $150 \mathrm{~mL}$ of $\mathrm{NaOH}(10.500 \mathrm{~g})$ aqueous solution, $120 \mathrm{~mL}$ of $\mathrm{CaCl}_{2}(3.330 \mathrm{~g})$ aqueous solution and $180 \mathrm{~mL}$ of $\mathrm{NaH}_{2} \mathrm{PO}_{4} \cdot 2 \mathrm{H}_{2} \mathrm{O}(9.360 \mathrm{~g})$ aqueous solution were separately added to a mixture of methanol $(60 \mathrm{~mL})$, oleic acid $(105 \mathrm{~mL})$ and deionized water $(135 \mathrm{~mL})$ under vigorous mechanical stirring in an ice-water bath. Then, the resulting mixture was transferred to a Teflon-lined stainless steel autoclave with a volume of 1 liter, sealed, and heated at $180{ }^{\circ} \mathrm{C}$ for $20 \mathrm{~h}$. After cooling to room temperature, the product was collected, and washed with absolute ethanol and deionized water several times, and dispersed in deionized water to form a homogeneous suspension for further use.

\subsection{Preparation of UHANWs/Col porous nanocomposite}

The biomimetic UHANWs/collagen (UHANWs/Col) porous nanocomposite with $66.7 \mathrm{wt} \%$ UHANWs was prepared by the freeze drying method. Firstly, a homogeneous collagen aqueous solution with a concentration of $0.04 \mathrm{~g} \mathrm{~g}^{-1}$ was obtained by mixing the lyophilized collagen sponge with deionized water under vigorous magnetic stirring at room temperature. Then, the as-prepared UHANWs aqueous suspension $\left(0.04 \mathrm{~g} \mathrm{~g}^{-1}\right)$ was added to the collagen aqueous solution $\left(0.04 \mathrm{~g} \mathrm{~g}^{-1}\right)$ with a weight ratio of $2: 1$. After vigorous stirring, the homogeneous mixture was transferred into a 48 -well plate, frozen at $-20^{\circ} \mathrm{C}$ for $36 \mathrm{~h}$, and freeze-dried at $-20{ }^{\circ} \mathrm{C}$. Finally, the lyophilized UHANWs/Col nanocomposite was chemically cross-linked in a mixture of $80 \mathrm{vol} \%$ ethanol and $20 \mathrm{vol} \%$ deionized water containing $20 \mathrm{mM}$ EDC and $8 \mathrm{mM}$ NHS for $24 \mathrm{~h} .{ }^{18,33,34}$ Then, the cross-linked UHANWs/Col porous nanocomposite was rinsed with ethanol and deionized water several times, and dried at $37^{\circ} \mathrm{C}$. Similarly, the pure collagen sample as the control sample was prepared under the same conditions but using the pure collagen aqueous solution. The as-prepared pure collagen sample and UHANWs/Col porous nanocomposite were sterilized with $29 \mathrm{kGy}$ of ${ }^{60} \mathrm{Co}$ radiation before use.

\subsection{Characterization}

Scanning electron microscopy (SEM) and transmission electron microscopy (TEM) images of the samples were recorded with the field-emission scanning electron microscope (Hitachi SU8220, S-3400, Japan) and a field-emission transmission electron microscope (JEOL JEM-2100F, Japan), respectively. The UHANWs powder, pure collagen sample, and biomimetic UHANWs/Col porous nanocomposite were compressed into tablets and characterized with X-ray diffraction (XRD) using an $\mathrm{X}$-ray diffractometer (Rigaku D/max $2550 \mathrm{~V}, \mathrm{Cu} \mathrm{K} \alpha$ radiation, $\lambda=$ $1.54178 \AA$ ). 


\subsection{Porosity of the as-prepared porous nanocomposite}

The porosity as the percentage of void space in a porous nanocomposite was calculated from the relative density value. ${ }^{22,35,36}$ The relative density $\left(\rho_{\mathrm{a}} / \rho_{\mathrm{m}}\right)$ was determined from the apparent density $\left(\rho_{\mathrm{a}}\right)$ and material density $\left(\rho_{\mathrm{m}}\right)$ of the freezedried porous nanocomposite. The material density of solid collagen and HAP was assumed to be 1.30 and $3.15 \mathrm{~g} \mathrm{~mL}^{-1}$, respectively. ${ }^{35,37}$ Thus, the material density of the collagen sample and UHANWs/Col porous nanocomposite with $66.7 \mathrm{wt} \%$ UHANWs was calculated to be 1.30 and $2.14 \mathrm{~g} \mathrm{~mL}^{-1}$, respectively. The apparent density of the freeze-dried porous nanocomposite was calculated using the equation $\left(\rho_{\mathrm{a}}=m / V\right)$, where $m$ and $V$ are the mass and volume of the freeze-dried porous nanocomposite, respectively. The porosity of the as-prepared freeze-dried porous nanocomposite was calculated using the formula:

$$
\text { Porosity }(\%)=\left(1-\rho_{\mathrm{a}} / \rho_{\mathrm{m}}\right) \times 100
$$

For each type of the nanocomposite, 5 samples were tested and the average value was reported.

\subsection{Mechanical properties of the nanocomposite}

The rehydrated porous nanocomposite was obtained by soaking the freeze-dried porous nanocomposite in deionized water for $12 \mathrm{~h}$. Then, the mechanical properties of the freeze-dried porous nanocomposite and rehydrated porous nanocomposite $(\Phi 10 \mathrm{~mm} \times 10 \mathrm{~mm})$ were characterized using a universal testing machine (Drick, China). The compressive modulus of the porous nanocomposite was calculated based on the slope of the stress-strain curve in the elastic strain within 0-10\% strain. For each type of the porous nanocomposite, 5 samples were tested and the average value was reported.

\subsection{In vitro degradation of the UHANWs/Col porous nanocomposite}

The release behaviors of $\mathrm{Ca}$ and $\mathrm{P}$ elements from the biomimetic UHANWs/Col porous nanocomposite were investigated as follows: the UHANWs/Col porous nanocomposite $(20 \mathrm{mg})$ was immersed in normal saline $(15 \mathrm{~mL})$, sealed, and shaken constantly $(120 \mathrm{rpm})$ at $37{ }^{\circ} \mathrm{C}$ in a desk-type constanttemperature oscillator (THI-92A, China). At given time intervals, $3 \mathrm{~mL}$ of the supernatant solution was withdrawn and measured by an inductively coupled plasma-optical emission spectrometer (ICP-OES, JY 2000-2, Horiba) to analyze the concentrations of $\mathrm{Ca}$ and $\mathrm{P}$ elements in the release medium, and replaced with the same volume of fresh normal saline.

\subsection{In vitro studies}

2.8.1. Cell isolation and culture. The rat bone marrowderived mesenchymal stem cells (rBMSCs) were isolated from the femurs of 4 week-old Sprague-Dawley (SD) rats. Firstly, the bone marrow of the diaphysis was flushed out and suspended in Dulbecco's modified Eagle's medium (DMEM, Gibco, USA) supplemented with $10 \%$ fetal bovine serum (Gibco, USA) and $1 \%(\mathrm{v} / \mathrm{v})$ penicillin/streptomycin (Gibco, USA). Then, the cells were cultured at $37{ }^{\circ} \mathrm{C}$ in a $5 \% \mathrm{CO}_{2}$ incubator, and the nonadherent cells were removed after $48 \mathrm{~h}$ of culture. The cells were subcultured at a confluence of $80-90 \%$, and the cells from passages 2 to 6 were used for the experiments.

2.8.2. Cell adhesion and morphology. The collagen sample and UHANWs/Col porous nanocomposite $(\Phi 10 \mathrm{~mm} \times 2 \mathrm{~mm})$ were placed into a 48 -well plate and seeded with $0.5 \mathrm{~mL}$ of cell suspension at a density of $1 \times 10^{5}$ cells per nanocomposite. After 3 days of culture, the cell morphologies on the collagen sample and UHANWs/Col porous nanocomposite were observed using a confocal laser scanning microscope (CLSM, LSM 510, Zeiss) and a scanning electron microscope (Hitachi S3400 , Japan). For the cytoskeleton staining, the porous nanocomposite with cells was fixed in $4 \%$ paraformaldehyde, treated with Triton X-100 (0.5 vol\%) for $5 \mathrm{~min}$, and stained with rhodamine phalloidin (Sigma) and 4',6-diamidino-2phenylindole (DAPI; Sigma) in darkness for $30 \mathrm{~min}$ and $5 \mathrm{~min}$, respectively. Then, the cells on the porous nanocomposite were observed and imaged with CLSM. For SEM observation, the porous nanocomposite with cells was fixed with $2.5 \mathrm{vol} \%$ glutaraldehyde for $4 \mathrm{~h}$, dehydrated in a series of ethanol solutions with gradient concentrations (50, 70, 80, 90, 95 , and $100 \mathrm{vol} \%$ ) for $10 \mathrm{~min}$, and freeze-dried. After coated with platinum, the cells on the porous nanocomposite were observed with SEM.

2.8.3. Cell proliferation. The proliferation of rBMSCs on the collagen sample and UHANWs/Col porous nanocomposite was assessed using the cell counting Kit-8 assay (CCK-8, Dojindo Molecular Technologies, Inc., Japan). Briefly, the rBMSCs were seeded on the porous nanocomposite $(\Phi 10 \mathrm{~mm}$ $\times 2 \mathrm{~mm}$ ) at a density of $1 \times 10^{5}$ cells per nanocomposite and cultured for 1,3 and 5 day(s). At each time point, the culture medium was removed and the cells were washed twice with phosphate buffered saline (PBS). Then, $500 \mu \mathrm{L}$ of fresh medium containing $10 \%$ CCK- 8 was added to each well. After incubation for $2 \mathrm{~h}$ at $37{ }^{\circ} \mathrm{C}$, aliquots $(100 \mu \mathrm{L})$ from each well were transferred to a 96-well plate for measurement. The absorbance of the samples was measured with a microplate reader (Bio-Rad 680 , USA) at $450 \mathrm{~nm}$. The result was expressed as the absorbance of the aliquot minus that of the well without cells, and each data was represented as the mean value of three parallel measurements.

\subsection{In vivo bone regeneration}

2.9.1. Animal surgical procedure. The animal experiments were performed in accordance with the Guide for the Care and Use of Laboratory Animals of National Research Council and approved by the Animal Research Ethics Committee of the Second Military Medical University. 24 New Zealand white rabbits $(2.5-3.0 \mathrm{~kg})$ were used in the animal experiments. The rabbits were randomly divided into the UHANWs/Col porous nanocomposite group $(n=12)$ and the collagen control group ( $n$ $=12$ ). After anesthetized with intravenous pentobarbital sodium (30 mg per $\mathrm{kg}$ body weight), a concave bone defect 
(15 $\mathrm{mm}$ long, $3 \mathrm{~mm}$ deep) was created at the middle segment of the radius of the right forelimb. Followed by a rinse of sterile physiological saline solution, the defect was implanted with the collagen or UHANWs/Col porous nanocomposite. Then, the incision was sutured using absorbable suture. After surgery, each rabbit was given penicillin injection.

2.9.2. Micro-computed tomography (micro-CT) assessment. The animals were sacrificed at 4,8 and 12 weeks postoperation (4 rabbits for each time point and each group). The bone specimens were collected and scanned by a micro-CT scanner (mCT-80, Scanco Medical, Switzerland) to evaluate the new bone formation in the defects. The morphologies of the bone specimens were reconstructed into 3-D images. New bone volume relative to tissue volume (BV/TV) and trabecular number (Tb.N) were quantified by micro-CT.

2.9.3. Histological analysis. For the histological analysis, the bone specimens were decalcified, dehydrated in ethanol solutions with ascending concentrations, and embedded in paraffin. Then, histological longitudinal sections ( $5 \mu \mathrm{m}$ thick) of each bone specimen were made in the middle part of the defects which were parallel to the long axis of the radius. The sections were stained with hematoxylin and eosin (H\&E) and observed with an optical microscope.

\subsection{Statistical analysis}

The data was presented as the mean \pm standard deviation (SD). One-way analysis of variance (ANOVA) and Student-NewmanKeuls post hoc tests were used to determine the level of significance. And the values of $p<0.05$ were accepted to be significant.

\section{Results and discussion}

\subsection{Morphological characterization of the as-prepared nanocomposite}

Ultralong hydroxyapatite nanowires (UHANWs) as the inorganic constituent of the as-prepared UHANWs/Col porous nanocomposite were synthesized by the calcium oleate precursor solvothermal method at $180{ }^{\circ} \mathrm{C}$ for $20 \mathrm{~h}$. As shown in Fig. 1, the as-prepared UHANWs have diameters of about 10-40 $\mathrm{nm}$ and ultralong lengths of several hundred micrometers. Moreover,
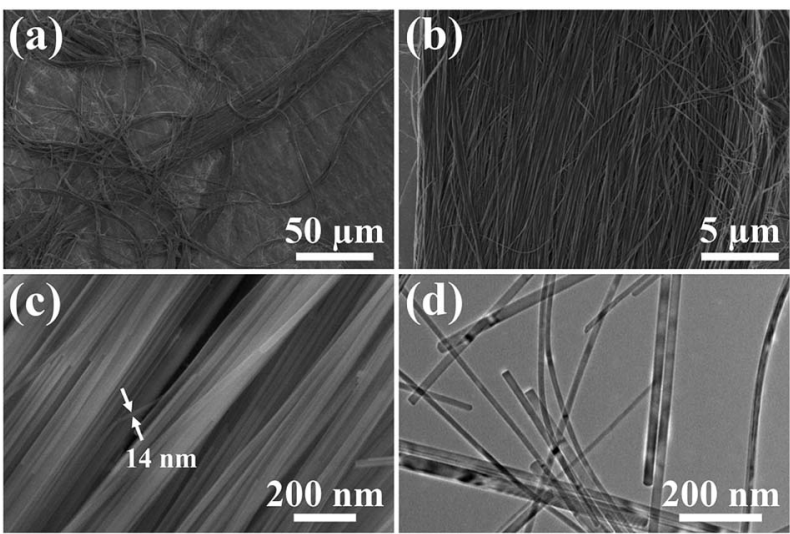

Fig. 1 SEM micrographs $(\mathrm{a}-\mathrm{c})$ and TEM image $(\mathrm{d})$ of the as-prepared highly flexible ultralong hydroxyapatite nanowires. the as-prepared UHANWs exhibit excellent flexibility and are bendable at an arbitrary degree. These experimental results demonstrate that the as-prepared UHANWs have obvious advantages in constructing hierarchical porous nanocomposite with enhanced mechanical properties.

In consideration of the chemical composition and structure of the natural bone, the biomimetic UHANWs/Col porous nanocomposite with 66.7 wt\% UHANWs was synthesized by freeze-drying the aqueous suspension containing UHANWs and collagen. From the insets in Fig. 2a and d, one can see that the as-prepared collagen sample and UHANWs/Col porous nanocomposite exhibit a cylindrical shape and are similar in size. The collagen sample shows pale yellow in color, whereas the UHANWs/Col porous nanocomposite exhibits milk-white in color owing to the presence of a high weight fraction of UHANWs. The structure of the collagen sample and UHANWs/ Col porous nanocomposite is evaluated by SEM (Fig. 2). As shown in Fig. 2a and d, the as-prepared collagen sample and UHANWs/Col nanocomposite are highly porous and exhibit a three-dimensional interconnected pore network with pore sizes ranging from 200 to $400 \mu \mathrm{m}$, which is beneficial for the flow transportation of nutrients, cell growth and bone ingrowth. ${ }^{38,39}$ Higher magnification SEM images further reveal that the surface of pore walls of the collagen sample is dense and relatively smooth (Fig. $2 \mathrm{~b}$ and c), whereas the surface of pore walls of the UHANWs/Col porous nanocomposite (Fig. 2e and f) is significantly different from the collagen sample, which is obviously rough. Moreover, the highly flexible UHANWs are uniformly covered by collagen and interweave with each other to form the hierarchical porous fabric-like structure.
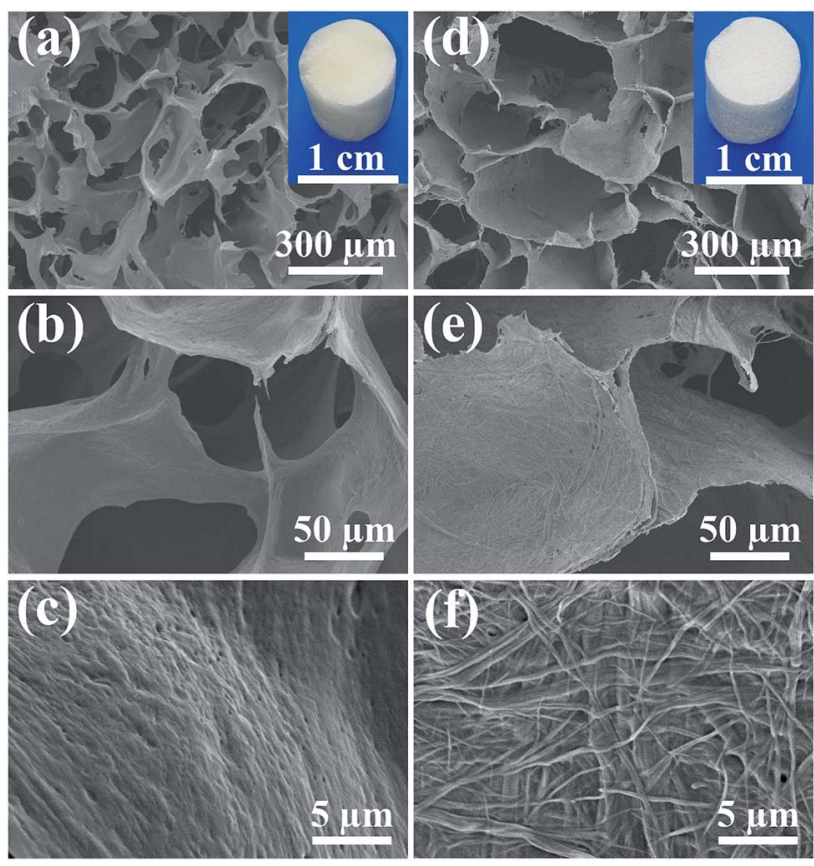

Fig. 2 SEM images: $(a-c)$ the collagen sample; $(d-f)$ the biomimetic UHANWs/Col porous nanocomposite with $66.7 \mathrm{wt} \%$ UHANWs. The insets in (a) and (d) are digital images of the collagen sample and the UHANWs/Col porous nanocomposite, respectively. 
3.2. Characterization of the chemical composition of the asprepared nanocomposite

The chemical compositions of the UHANWs, collagen sample, and UHANWs/Col nanocomposite were further analyzed by XRD. As shown in Fig. 3, the XRD pattern of the collagen sample exhibits the characteristic peaks of collagen with a weak diffraction peak at around $8^{\circ}$ and a broad hump at around $22^{\circ}{ }^{\mathbf{1 8 , 4 0}}$ indicating that the collagen sample has a low crystallinity. The well-defined diffraction peaks of UHANWs at $2 \theta$ values of $10.8^{\circ}, 18.8^{\circ}, 21.8^{\circ}, 29.0^{\circ}, 31.8^{\circ}, 32.9^{\circ}, 39.8^{\circ}, 48.6^{\circ}$, $51.3^{\circ}$ and $59.9^{\circ}$ correspond to the (100), (110), (200), (210), (211), (300), (310), (320), (410) and (420) crystal planes of HAP, the crystal phase of the as-prepared UHANWs can be indexed to a single phase of HAP with a hexagonal structure $\left(\mathrm{Ca}_{10}\left(\mathrm{PO}_{4}\right)_{6}(-\right.$ $\mathrm{OH})_{2}$, JCPDS no. 09-0432). ${ }^{18,27}$ Moreover, the XRD pattern of the UHANWs/Col porous nanocomposite exhibits both the characteristic peaks of collagen and HAP. The as-prepared biomimetic UHANWs/Col porous nanocomposite with $66.7 \mathrm{wt} \%$ UHANWs is promising for the applications in various biomedical fields such as bone defect repair. ${ }^{41-43}$

\subsection{Porosity and pore interconnectivity of the as-prepared nanocomposite}

Scaffolds with porous structure and well-interconnected large pores can support and promote cell infiltration and neovascularization, which is beneficial for the new bone ingrowth. ${ }^{23-25}$ The porosity and pore interconnectivity of the asprepared biomimetic UHANWs/Col porous nanocomposite with 66.7 wt $\%$ UHANWs were further investigated. As shown in Fig. 4, the collagen sample and UHANWs/Col nanocomposite are highly porous and exhibit the similar porosity of $96.56 \pm$ 0.48 and $96.61 \pm 0.22 \%$, respectively, which is in the porosity range of the human cancellous bone. ${ }^{44}$ In addition, the high porosities of the collagen sample and UHANWs/Col nanocomposite are consistent with the SEM observation of the porous structure of the nanocomposite (Fig. 2).

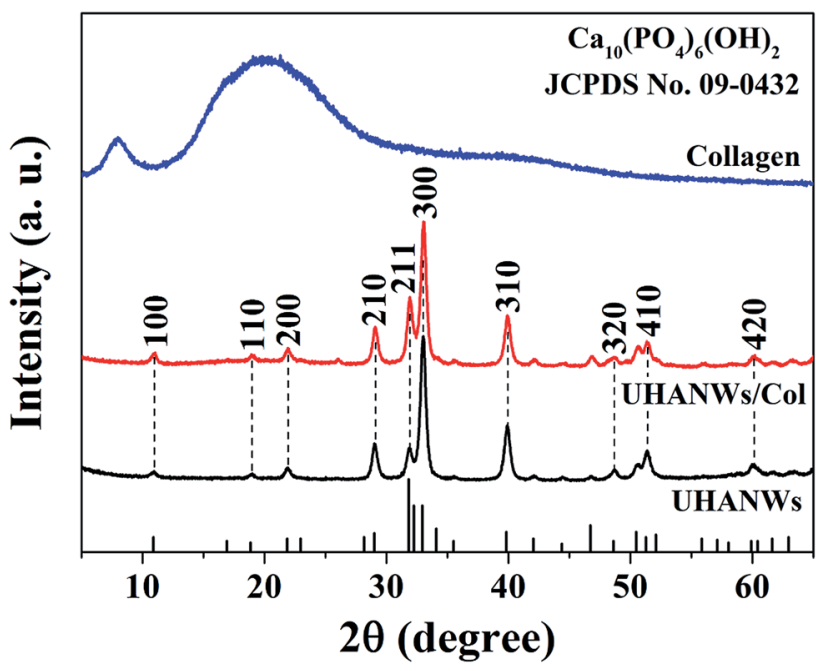

Fig. 3 XRD patterns of the collagen sample, UHANWs, and UHANWs/ Col porous nanocomposite.

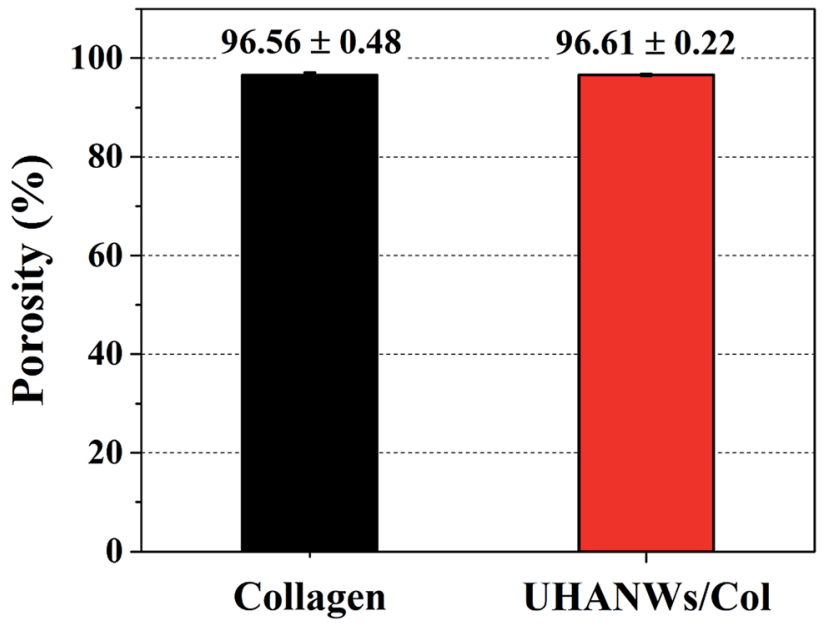

Fig. 4 Porosities of the collagen sample and biomimetic UHANWs/ Col porous nanocomposite with 66.7 wt\% UHANWs.

To demonstrate the porous structure and excellent pore interconnectivity of the as-prepared biomimetic UHANWs/Col porous nanocomposite, several drops of alamarBlue cell viability assay reagent were dropped on the top surface of the UHANWs/Col porous nanocomposite (Fig. 5). The alamarBlue cell viability assay reagent is quickly absorbed by the UHANWs/Col porous nanocomposite, and the top half of the nanocomposite becomes dark-blue (Fig. 5b). The alamarBlue cell viability assay reagent gradually permeates to the bottom half of the UHANWs/Col porous nanocomposite (Fig. 5c), then the whole UHANWs/Col porous nanocomposite becomes dark-blue (Fig. 5d and e). After cutting the UHANWs/Col porous nanocomposite open, one can see that the interior of the UHANWs/Col porous nanocomposite is evenly dyed dark-blue and exhibits highly porous structure with a large number of macroscopical pores (Fig. 5f).

To further investigate the pore interconnectivity of the biomimetic UHANWs/Col porous nanocomposite, the UHANWs/Col porous nanocomposite was rehydrated in deionized water and placed in a glass Petri dish with 5 vol\% alamarBlue cell viability assay reagent solution. When contacting with the alamarBlue cell viability assay reagent solution, the bottom half of the hydrated UHANWs/Col porous nanocomposite becomes blue rapidly (Fig. 6b). After being squeezed and released, the whole UHANWs/Col porous nanocomposite becomes blue rapidly (Fig. 6c-e), indicating that the alamarBlue cell viability assay reagent solution goes from the bottom to the top of the UHANWs/Col porous nanocomposite, which can be explained by its excellent pore interconnectivity and high hydrophilicity. These experimental results further confirm that the biomimetic UHANWs/Col porous nanocomposite is highly porous with well-interconnected pores. Moreover, the excellent elastic property reveals the excellent mechanical properties of the as-prepared UHANWs/Col porous nanocomposite.

\subsection{Mechanical properties of the as-prepared nanocomposite}

The mechanical properties of the implanted scaffolds play an important role in bone regeneration and reconstruction. For 


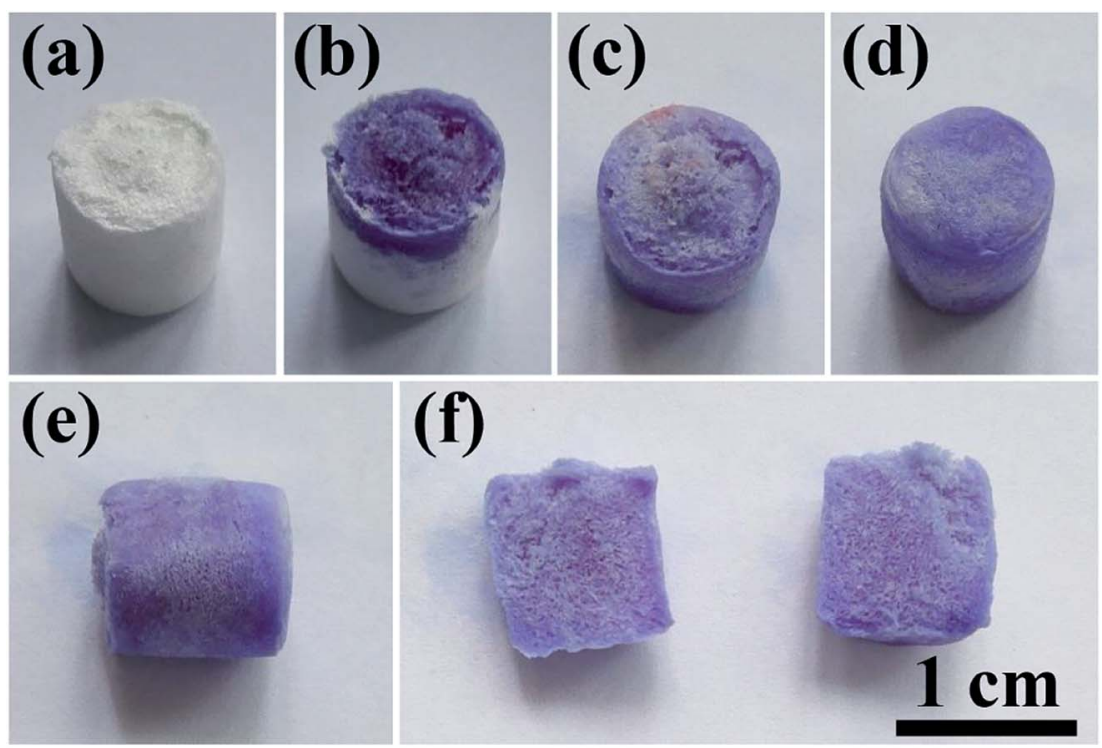

Fig. 5 Characterization of the porous structure and pore interconnectivity of the biomimetic UHANWs/Col porous nanocomposite with 66.7 wt\% UHANWs: (a) digital image of the UHANWs/Col porous nanocomposite; $(b-e)$ the permeable performance of the alamarBlue cell viability assay reagent from the top to the bottom of the UHANWs/Col porous nanocomposite; (f) digital images in the cross section from top to bottom of the UHANWs/Col porous nanocomposite.

instance, the scaffolds with rigid matrices could significantly stimulate the expression of osteogenesis-related genes of mesenchymal stem cells via the mechanical signaling pathways. ${ }^{9,45,46}$ In addition, the implanted scaffolds provide sufficient mechanical strength and mechanical stability in vivo to withstand mechanical loading and support the new formed bone. ${ }^{7,47}$ In this work, the compressive mechanical properties of the collagen sample and biomimetic UHANWs/Col porous nanocomposite were investigated under dry and rehydrated states, as shown in Fig. 7. The addition of UHANWs has an obvious influence on the mechanical properties of the collagen sample (Fig. 7a and b). Compared with the collagen sample, the biomimetic UHANWs/Col porous nanocomposite exhibits significantly enhanced mechanical properties both under dry and rehydrated conditions (Fig. 7c and d). Moreover, the compressive modulus of the UHANWs/Col porous nanocomposite in a dry state $(839.4 \pm 66.9 \mathrm{kPa})$ and rehydrated state $(76.06 \pm 7.27 \mathrm{kPa})$ is about 7 and 4 times those of the collagen sample $(116.3 \pm 19.6 \mathrm{kPa}$ and $18.32 \pm 2.25 \mathrm{kPa})$, respectively, indicating that the UHANWs are the promising reinforcing material and can remarkably improve the mechanical properties of the

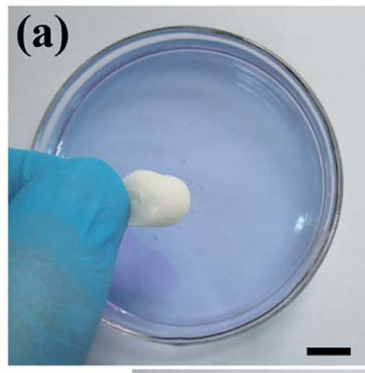

(d)

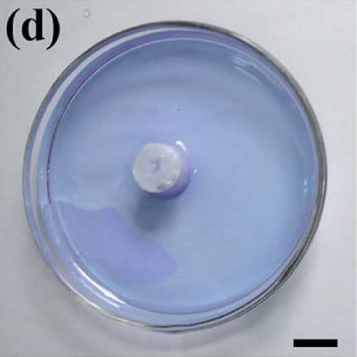

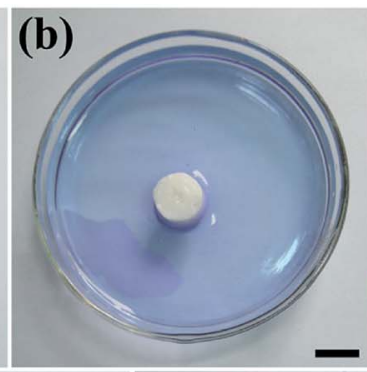

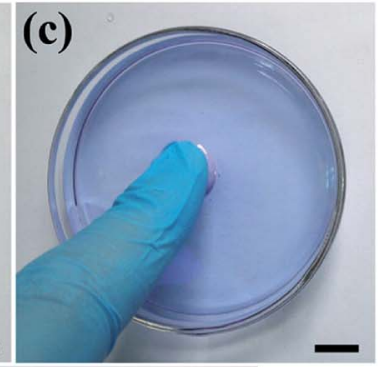

(e)

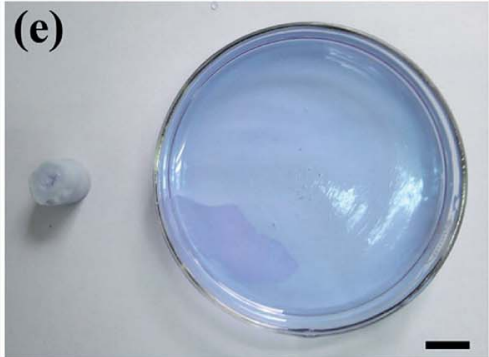

Fig. 6 Excellent pore interconnectivity of the biomimetic UHANWs/Col porous nanocomposite with 66.7 wt\% UHANWs: (a and b) the rehydrated UHANWs/Col porous nanocomposite is placed in a glass Petri dish with 5 vol\% alamarBlue cell viability assay reagent solution; (c) squeezing the UHANWs/Col porous nanocomposite with a finger; ( $d$ and e) releasing the UHANWs/Col porous nanocomposite after squeezing. The scale bar represents $1 \mathrm{~cm}$. 

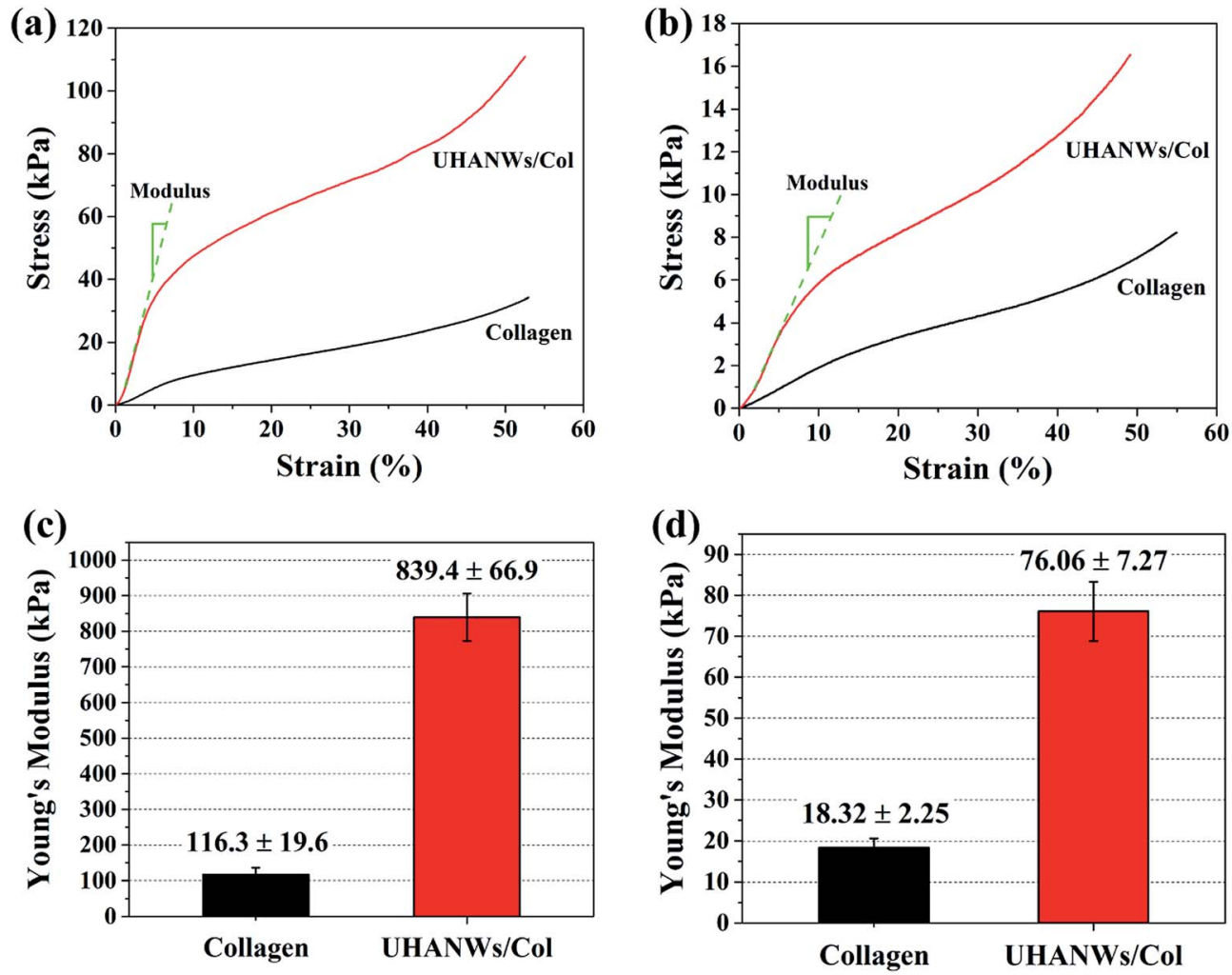

Fig. 7 Compressive mechanical properties of the collagen sample and biomimetic UHANWs/Col porous nanocomposite with 66.7 wt\% UHANWs: Stress-strain curves ( $a$ and b) and Young's modulus ( $c$ and $d$ ) of the freeze-dried porous nanocomposite (a and $c$ ) and rehydrated porous nanocomposite (b and $d$ ).

UHANWs/Col nanocomposite while retaining the highly porous and interconnected pore structure of the nanocomposite.

The excellent mechanical properties of the biomimetic UHANWs/Col porous nanocomposite with $66.7 \mathrm{wt} \%$ UHANWs were further investigated. As shown in Fig. 8 and Video S1 in the $\mathrm{ESI}, \uparrow$ the rehydrated UHANWs/Col porous nanocomposite can be repeatedly squeezed and rapidly rebound to its original shape, indicating excellent elastic property of the as-prepared biomimetic UHANWs/Col porous nanocomposite. During the repeating compression-release-compression-release cycles, the UHANWs/Col porous nanocomposite undergoes a reversible geometry change with an excellent elastic performance. When the porous UHANWs/Col nanocomposite is compressed, the internal pore walls and channels of the UHANWs/Col nanocomposite are deformed but not damaged. When the compression force is removed, the deformed pore walls and channels expand and restore the original shape owing to excellent mechanical properties and absorption of water.

The significantly improved mechanical properties of the asprepared UHANWs/Col porous nanocomposite mainly results from the biomimetic porous structure constructed with the highly flexible UHANWs and collagen matrix. The abundant -OH groups of UHANWs can form hydrogen bonds with the $-\mathrm{OH}$ groups and $-\mathrm{NH}_{2}$ groups of collagen matrix. ${ }^{48}$ The calcium ions on the terminated surface of hydroxyapatite crystals may form the bond with the end groups $-\mathrm{NH}_{2}$ and $-\mathrm{COOH}$ of collagen matrix. ${ }^{49}$ In addition, the collagen triple helix can entangle with the UHANWs. ${ }^{18}$ Thus, the highly flexible UHANWs can form the UHANW-collagen nanocomposite fibrils. The UHANW-collagen nanocomposite fibrils exhibit high flexibility and further interweave with each other to
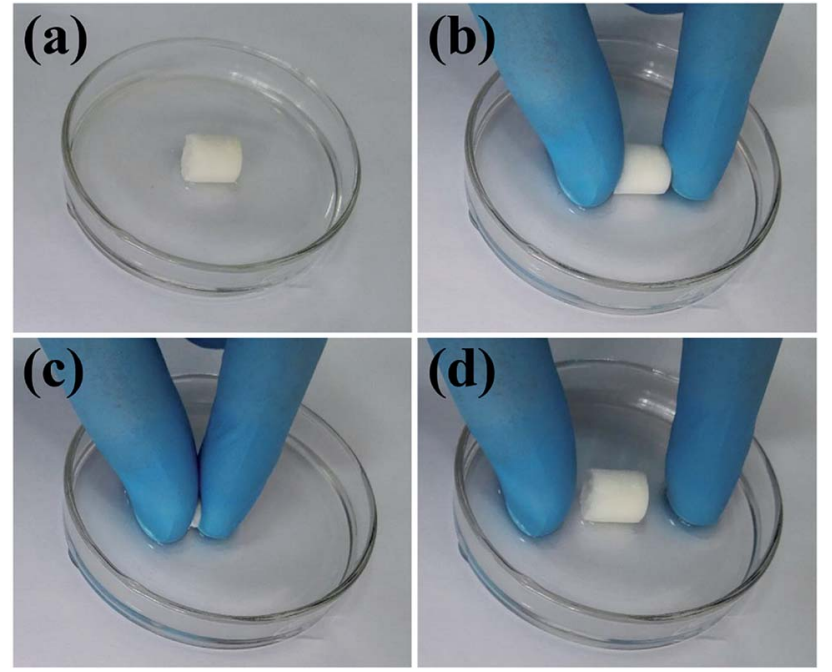

Fig. 8 Mechanical properties of the biomimetic UHANWs/Col porous nanocomposite with 66.7 wt\% UHANWs: (a) digital image of the UHANWs/Col porous nanocomposite rehydrated in deionized water; $(b-d)$ the rehydrated UHANWs/Col porous nanocomposite can be repeatedly squeezed ( $b$ and $c$ ) and rebound rapidly to its original cylindrical shape (d). 
construct the three-dimensional biomimetic porous structure of the UHANWs/Col nanocomposite, which endows the UHANWs/Col porous nanocomposite with significantly enhanced mechanical properties.

\subsection{In vitro degradation of the UHANWs/Col porous nanocomposite}

In consideration of the superior mechanical properties, the biomimetic UHANWs/Col porous nanocomposite with $66.7 \mathrm{wt} \%$ UHANWs has a promising potential for bone tissue engineering applications. Thus, the degradation behavior of the as-prepared UHANWs/Col porous nanocomposite was further assessed by investigating the release performance of $\mathrm{Ca}$ and $\mathrm{P}$ elements in aqueous solution. As shown in Fig. 9, Ca and P elements exhibit similar release profiles from the biomimetic UHANWs/Col porous nanocomposite in the normal saline. The release of $\mathrm{Ca}$ and $\mathrm{P}$ elements is rapid in the first 5 days, then slows down, and reaches a plateau. After soaking in the normal saline for 21 days, the cumulative release amounts of $\mathrm{Ca}$ and $\mathrm{P}$ elements are $53.9 \%$ and $36.1 \%$, respectively. These experimental results demonstrate that the as-prepared biomimetic UHANWs/Col porous nanocomposite has good degradation properties.

\subsection{Cell adhesion and proliferation on the UHANWs/Col porous nanocomposite}

The biocompatibility of the UHANWs/Col porous nanocomposite with 66.7 wt\% UHANWs was assessed by the cell adhesion and proliferation tests. After 3 days of culture, the rBMSCs cells maintain their phenotype and spread well with filopodia on the surface of the collagen sample and UHANWs/Col porous nanocomposite (Fig. 10). More significantly, the rBMSCs on the surface of the UHANWs/Col porous nanocomposite (Fig. 10d-f) exhibit prominent cytoplasmic extension and spread well with filopodia and lamellipodia compared with those on the collagen

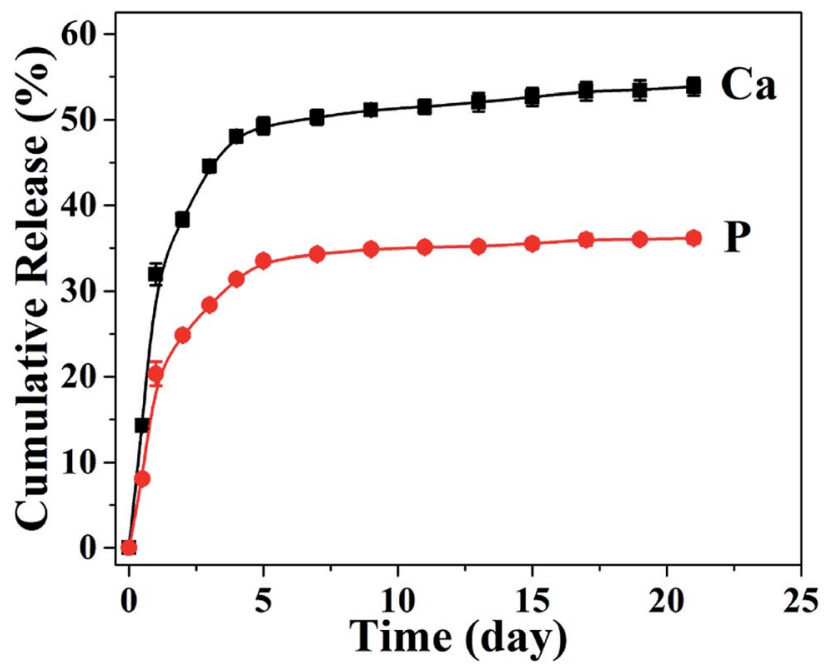

Fig. 9 The release profiles of $\mathrm{Ca}$ and $\mathrm{P}$ elements from the biomimetic UHANWs/Col porous nanocomposite after soaking in the normal saline for different times.
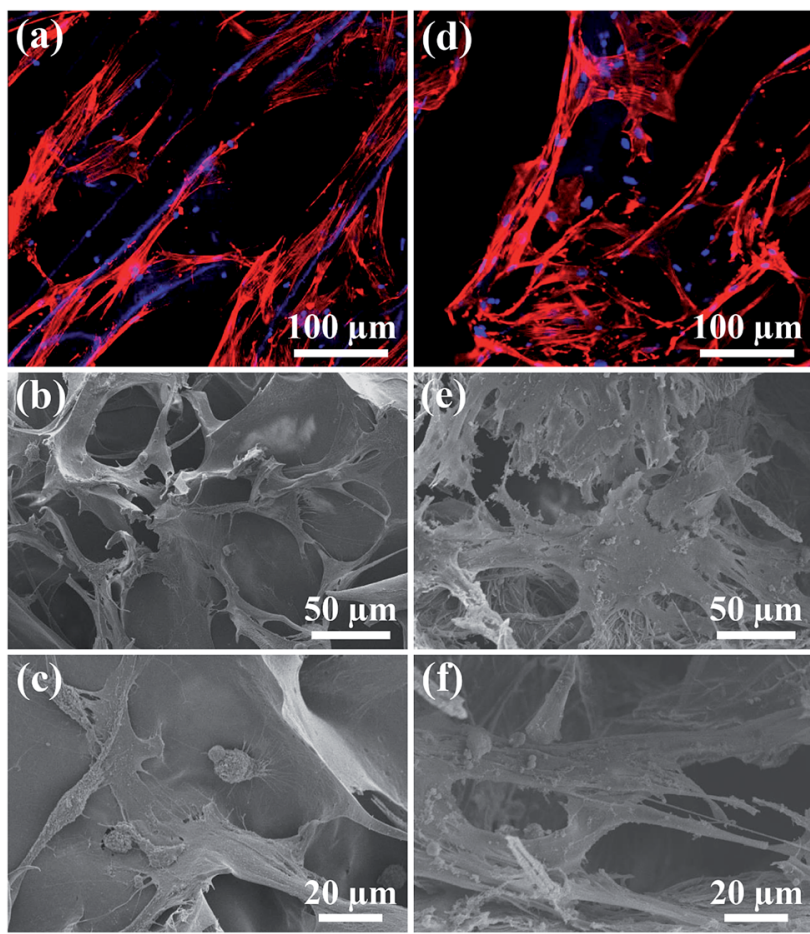

Fig. 10 Cell morphology observation. (a and d) Cytoskeleton staining; (b, c, e and f) SEM micrographs of rBMSCs after culture for 3 days on the collagen sample $(a-c)$ and biomimetic UHANWs/Col porous nanocomposite $(d-f)$.

sample (Fig. 10a-c), indicating that the UHANWs/Col porous nanocomposite is beneficial to cell attachment.

The proliferation of rBMSCs on the collagen sample and UHANWs/Col porous nanocomposite was determined by the CCK-8 assay, and the experimental results are shown in Fig. 11. The collagen sample and UHANWs/Col porous nanocomposite exhibit a good biocompatibility and are favorable for the proliferation of rBMSCs in the first 5 days of culture, then the cell proliferation on the collagen sample slows down, and the

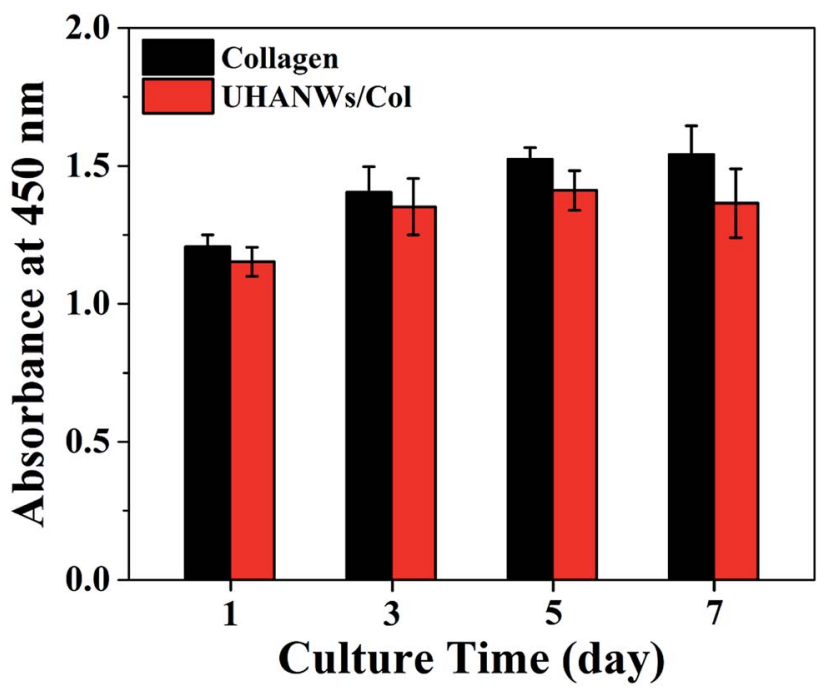

Fig. 11 The CCK-8 assay of the rBMSCs proliferation on the collagen sample and biomimetic UHANWs/Col porous nanocomposite. 


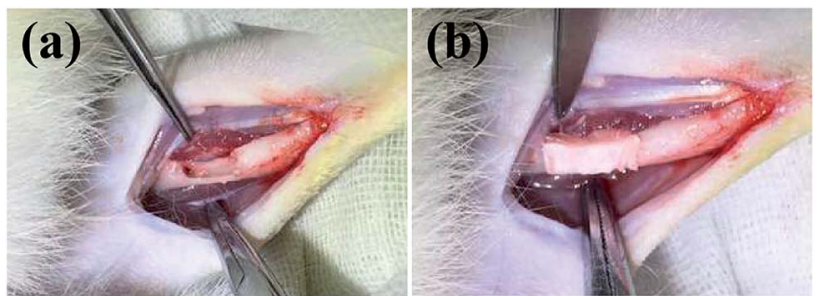

Fig. 12 (a) Concave bone defect ( $15 \mathrm{~mm}$ long, $3 \mathrm{~mm}$ deep) of the rabbit adialis defect region; (b) implantation of the biomimetic UHANWs/ Col porous nanocomposite ( $15 \mathrm{~mm}$ long, $3 \mathrm{~mm}$ wide, $3 \mathrm{~mm}$ thick).

cell number on the UHANWs/Col porous nanocomposite decreases slightly. The reduced cell number on the UHANWs/ Col porous nanocomposite may be explained by the ioninduced effect due to the excessive calcium and phosphate ions released from the UHANWs/Col porous nanocomposite, leading to the change of the $\mathrm{pH}$ value of the surrounding culture medium. However, this effect can be minimized in vivo..$^{34,50}$

\subsection{In vivo bone regeneration}

The bone defect repairing ability of the biomimetic UHANWs/ Col porous nanocomposite was assessed by implanting the nanocomposite into a rabbit radialis defect model (Fig. 12). At 4, 8 and 12 weeks post-implantation, the formation of new bone in the radialis defect region was evaluated by micro-CT (Fig. 13) and histological analysis (Fig. 14). As shown in Fig. 13a, the three-dimensional (3-D) images of the rabbit radialis defect region at different times after implantation were reconstructed. With increasing implantation time, more new bone formation is observed in the radialis defect region of the collagen sample group or UHANWs/Col nanocomposite group. Compared with the collagen control group, much more new bone is formed in the UHANWs/Col nanocomposite group. Most importantly, a relatively normal bone marrow cavity is formed in the UHANWs/Col nanocomposite group after 12 weeks postimplantation. By contrast, the bone defect repairing performance of the collagen control group is not satisfactory, resulting in the formation of an irregular bone marrow cavity at 12 weeks post-implantation.

To evaluate bone regeneration inside the porous nanocomposite, the quantitative analysis of the rabbit radialis defect region of the collagen control group and biomimetic UHANWs/ Col nanocomposite group were further assessed. For the collagen control group at 4, 8 and 12 weeks post-implantation, the percentage of new bone volume to tissue volume (BV/TV) is (a)

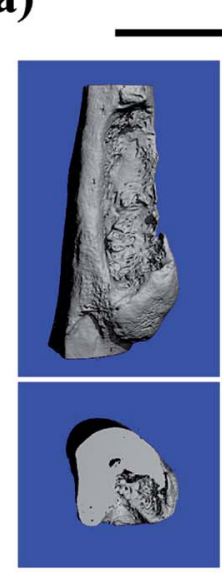

4 Weeks
Collagen
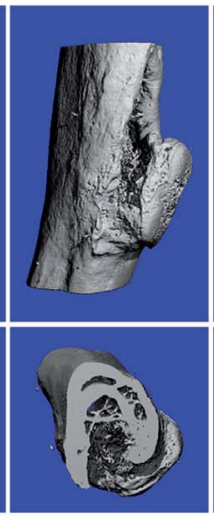

8 Weeks

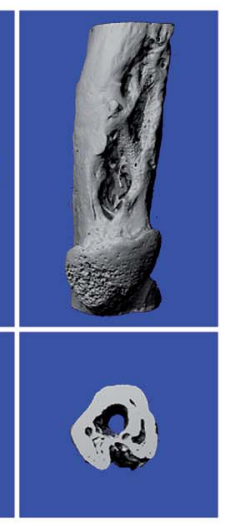

12 Weeks

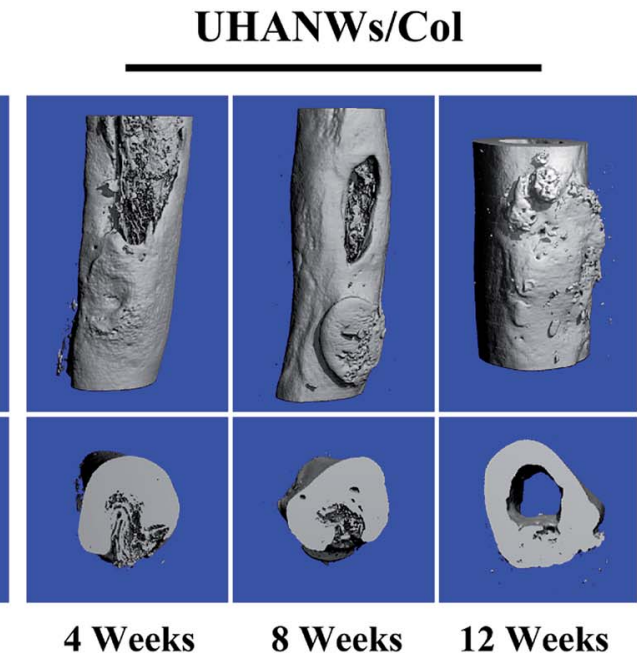

(b)

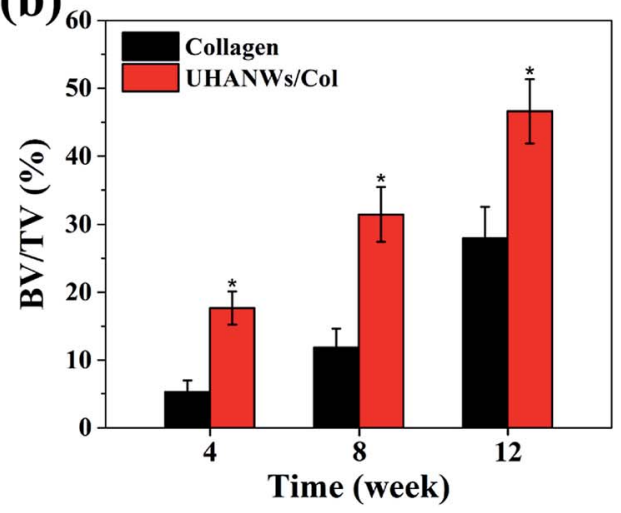

(c)

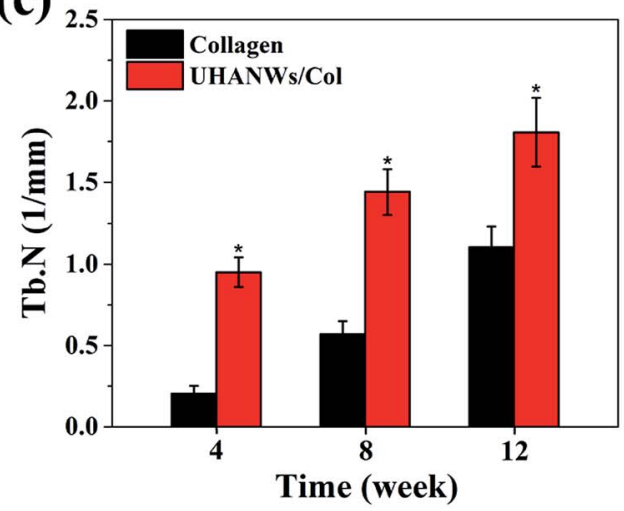

Fig. 13 Micro-CT analysis of newly formed bone in rabbit radialis defect region implanted with the collagen sample and UHANWs/Col porous nanocomposite for 4, 8 and 12 weeks post-implantation: (a) three-dimensional (3-D) reconstructed rabbit radialis images; (b) the percentage of new bone volume to tissue volume (BV/TV); (c) trabecular number (Tb.N) in the defects. *, p < 0.05 compared with the collagen sample. 


\section{Collagen}
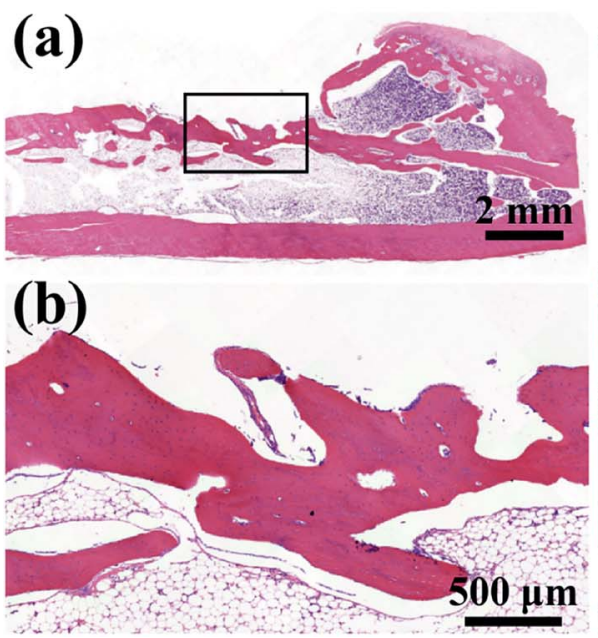

\section{UHANWs/Col}
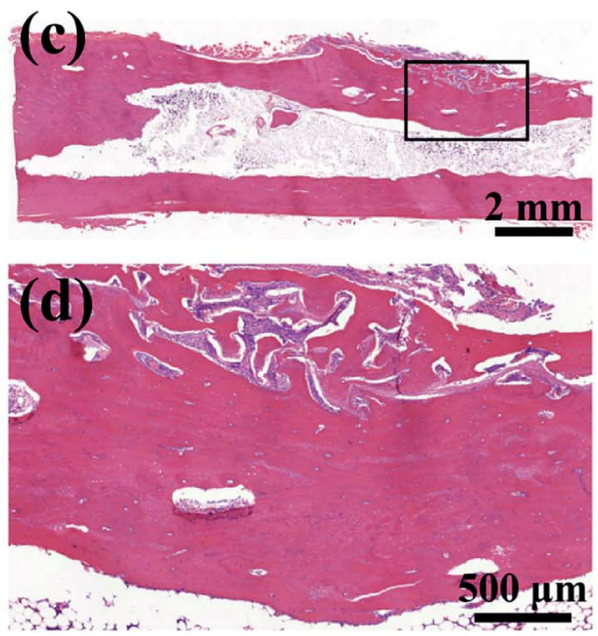

Fig. 14 Histological analysis of bone regeneration. Hematoxylin and eosin stained section of rabbit radialis defect region implanted for 12 weeks with the collagen sample ( $a$ and $b$ ) and UHANWs/Col porous nanocomposite ( $c$ and d).

$5.24 \pm 1.73 \%, 11.86 \pm 2.81 \%$, and $27.95 \pm 4.59 \%$, respectively (Fig. 13b), the corresponding trabecular number (Tb.N) is 0.20 $\pm 0.05 \mathrm{~mm}^{-1}, 0.57 \pm 0.08 \mathrm{~mm}^{-1}$, and $1.10 \pm 0.13 \mathrm{~mm}^{-1}$, respectively (Fig. 13c). More significantly, after 4, 8 and 12 weeks post-implantation, the $\mathrm{BV} / \mathrm{TV}$ in the UHANWs/Col nanocomposite group is $17.67 \pm 2.45 \%, 31.46 \pm 4.01 \%$, and $46.61 \pm$ $4.74 \%$, respectively (Fig. 13b), and the corresponding Tb.N in the UHANWs/Col nanocomposite group is $0.95 \pm 0.09 \mathrm{~mm}^{-1}$, $1.44 \pm 0.14 \mathrm{~mm}^{-1}$, and $1.81 \pm 0.21 \mathrm{~mm}^{-1}$ (Fig. 13c), respectively, which are significantly higher $(p<0.05)$ than those in the collagen control group. These experimental results demonstrate that the biomimetic UHANWs/Col porous nanocomposite can significantly enhance bone regeneration in vivo compared with the collagen sample.

The new bone formation in the rabbit radialis defect region was further assessed by histological analysis. Hematoxylin and eosin staining of the longitudinal section in the middle part of the bone defect with the porous nanocomposite implanted for 12 weeks is shown in Fig. 14. In the collagen sample group and UHANWs/Col nanocomposite group, the remnant of the nanocomposite is still present in the defect region and surrounded by the newly formed bone. Moreover, the newly formed bone tissues wrap around the implanted nanocomposite and grow throughout the entire nanocomposite. By contrast, much more new bone tissues are observed within the defect region of the UHANWs/Col nanocomposite group compared with those of the collagen control group, which is consistent with the micro-CT analysis (Fig. 13). Most importantly, the woven bone and lamellar bone are formed along the long axis of the radialis defect and tightly integrate with the UHANWs/Col porous nanocomposite, and further connect directly with the host bone to construct a relatively normal bone marrow cavity (Fig. 14c and d), indicating that the as-prepared biomimetic UHANWs/ Col porous nanocomposite can significantly improve bone regeneration and bone integration in vivo compared with the collagen sample.
Compared with the collagen sample, the biomimetic UHANWs/Col porous nanocomposite can remarkably facilitate the formation of new bone in vivo, which can be explained by the presence of UHANWs and the biomimetic porous structure of the nanocomposite. On the one hand, the addition of UHANWs can significantly enhance the mechanical properties of the nanocomposite, which is beneficial to the bone-related gene expression of mesenchymal stem cells via the mechanical signaling pathways. ${ }^{45,46,51}$ In addition, the $\mathrm{Ca}$ and $\mathrm{P}$ elements released from the UHANWs/Col porous nanocomposite can provide a positive chemical environment for the stem cell niche and stimulate the osteogenic responses of stem cells. ${ }^{9,52}$ The highly porous structure and well-interconnected large pores can promote flow transport of nutrients, cell infiltration, new bone ingrowth and bone integration. ${ }^{\mathbf{8 2 3 - 2 5}}$ More significantly, the biomimetic hierarchical pore walls of the UHANWs/Col nanocomposite constructed with the UHANWcollagen nanocomposite fibrils can mimic the human osteogenic niche and induce the osteogenic differentiation of stem cells, and further promote bone regeneration in vivo. ${ }^{\mathbf{1 3 , 4 2}}$ Furthermore, the biomimetic UHANWs/Col porous nanocomposite exhibits excellent mechanical properties and can provide mechanical support for the host bone and newly formed bone. Thus, the biomimetic UHANWs/Col porous nanocomposite with $66.7 \mathrm{wt} \%$ UHANWs is promising for the applications in various biomedical fields such as bone defect repair.

\section{Conclusions}

In this study, a biomimetic UHANWs/Col elastic porous nanocomposite with 66.7 wt\% UHANWs has been developed using ultralong hydroxyapatite nanowires and collagen by a freeze drying process and further chemical crosslinking. The asprepared UHANWs/Col porous nanocomposite has a highly porous structure, well-interconnected pores and hierarchical 
rough pore surface. Moreover, UHANWs can significantly enhance the mechanical properties of the UHANWs/Col porous nanocomposite. More significantly, the rehydrated UHANWs/ Col porous nanocomposite exhibits an excellent elastic performance, and can be repeatedly compressed and released and restore its original shape. Furthermore, the biomimetic UHANWs/Col porous nanocomposite exhibits good degradation properties with a sustained release of $\mathrm{Ca}$ and $\mathrm{P}$ elements, and can provide excellent microenvironment for adhesion and spreading of mesenchymal stem cells. In vivo evaluation demonstrates that the biomimetic UHANWs/Col porous nanocomposite can significantly enhance bone regeneration and bone integration compared with the collagen sample. After 12 weeks post-implantation, the woven bone and lamellar bone are formed throughout the entire UHANWs/Col porous nanocomposite and connect directly with the host bone to construct a relatively normal bone marrow cavity, leading to successful osteointegration and bone reconstruction. The biomimetic UHANWs/Col porous nanocomposite with $66.7 \mathrm{wt} \%$ UHANWs is promising for applications in various biomedical fields such as bone defect repair.

\section{Conflicts of interest}

There are no conflicts to declare.

\section{Acknowledgements}

Financial support from the Science and Technology Commission of Shanghai Municipality (15JC1491001), the National Natural Science Foundation of China (51472259) is gratefully acknowledged. Authors thank Zhuyun Cai for the help with animal experiments.

\section{References}

1 B. M. Chesnutt, Y. L. Yuan, K. Buddington, W. O. Haggard and J. D. Bumgardner, Tissue Eng., Part A, 2009, 15, 25712579.

2 R. Dimitriou, E. Jones, D. McGonagle and P. V. Giannoudis, BMC Med., 2011, 9, 66.

3 H. H. Liu, H. J. Peng, Y. Wu, C. Zhang, Y. Z. Cai, G. W. Xu, Q. Li, X. Chen, J. F. Ji, Y. Z. Zhang and H. W. OuYang, Biomaterials, 2013, 34, 4404-4417.

4 Z. Jamalpoor, H. Mirzadeh, M. T. Joghataei, D. Zeini, S. Bagheri-Khoulenjani and M. R. Nourani, J. Biomed. Mater. Res., Part A, 2015, 103, 1882-1892.

5 C. Mauffrey, M. Madsen, R. J. Bowles and D. Seligson, Injury, 2012, 43, 323-326.

6 S. Yunoki, H. Sugiura, T. Ikoma, E. Kondo, K. Yasuda and J. Tanaka, Biomed. Mater., 2011, 6, 015012.

7 P. Gupta, M. Adhikary, M. J. Christakiran, M. Kumar, N. Bhardwaj and B. B. Mandal, ACS Appl. Mater. Interfaces, 2016, 8, 30797-30810.

8 H. Jiang, Y. Zuo, Q. Zou, H. A. Wang, J. J. Du, Y. B. Li and X. C. Yang, ACS Appl. Mater. Interfaces, 2013, 5, 12036-12044.
9 T. W. Sun, W. L. Yu, Y. J. Zhu, R. L. Yang, Y. Q. Shen, D. Y. Chen, Y. H. He and F. Chen, ACS Appl. Mater. Interfaces, 2017, 9, 16435-16447.

10 J. J. Li, D. L. Kaplan and H. Zreiqat, J. Mater. Chem. B, 2014, 2, 7272-7306.

11 M. J. Meagher, H. E. Weiss-Bilka, M. E. Best, J. D. Boerckel, D. R. Wagner and R. K. Roeder, J. Biomed. Mater. Res., Part A, 2016, 104, 2178-2188.

12 G. M. Calori, E. Mazza, M. Colombo and C. Ripamonti, Injury, 2011, 42, S56-S63.

13 S. Minardi, B. Corradetti, F. Taraballi, M. Sandri, J. Van Eps, F. J. Cabrera, B. K. Weiner, A. Tampieri and E. Tasciotti, Biomaterials, 2015, 62, 128-137.

14 P. V. Giannoudis, J. J. C. Arts, G. Schmidmaier and S. Larsson, Injury, 2011, 42, S1-S2.

15 J. Y. Rho, L. Kuhn-Spearing and P. Zioupos, Med. Eng. Phys., 1998, 20, 92-102.

16 C. Qi, Y. J. Zhu, B. Q. Lu, X. Y. Zhao, J. Zhao and F. Chen, J. Mater. Chem., 2012, 22, 22642-22650.

17 L. P. Jia, Z. G. Duan, D. D. Fan, Y. Mi, J. F. Hui and L. Chang, Mater. Sci. Eng., C, 2013, 33, 727-734.

18 T. W. Sun, Y. J. Zhu, F. Chen and Y. G. Zhang, Chem.-Asian J., 2017, 12, 655-664.

19 L. Ma, C. Y. Gao, Z. W. Mao, J. Zhou, J. C. Shen, X. Q. Hu and C. M. Han, Biomaterials, 2003, 24, 4833-4841.

20 J. Glowacki and S. Mizuno, Biopolymers, 2008, 89, 338-344. 21 C. J. Dong and Y. G. Lv, Polymers, 2016, 8, 42.

22 R. J. Kane, H. E. Weiss-Bilka, M. J. Meagher, Y. X. Liu, J. A. Gargac, G. L. Niebur, D. R. Wagner and R. K. Roeder, Acta Biomater., 2015, 17, 16-25.

23 M. F. Zhu, K. Wang, J. J. Mei, C. Li, J. M. Zhang, W. T. Zheng, D. An, N. N. Xiao, Q. Zhao, D. L. Kong and L. Y. Wang, Acta Biomater., 2014, 10, 2014-2023.

24 Y. K. Dai, G. Liu, L. Ma, D. A. Wang and C. Y. Gao, J. Mater. Chem. B, 2016, 4, 4410-4419.

25 C. M. Murphy, M. G. Haugh and F. J. O'Brien, Biomaterials, 2010, 31, 461-466.

26 C. M. Tierney, M. G. Haugh, J. Liedl, F. Mulcahy, B. Hayes and F. J. O'Brien, J. Mech. Behav. Biomed. Mater., 2009, 2, 202-209.

27 T. W. Sun, Y. J. Zhu and F. Chen, Chem.-Eur. J., 2017, 23, 3850-3862.

28 Y. G. Zhang, Y. J. Zhu, F. Chen and J. Wu, Mater. Lett., 2015, 144, 135-137.

29 B. Q. Lu, Y. J. Zhu and F. Chen, Chem.-Eur. J., 2014, 20, 12421246.

30 Y. Y. Jiang, Y. J. Zhu, F. Chen and J. Wu, Ceram. Int., 2015, 41, 6098-6102.

31 F. Chen and Y. J. Zhu, ACS Nano, 2016, 10, 11483-11495.

32 T. W. Sun, Y. J. Zhu, F. Chen, F. F. Chen, Y. Y. Jiang, Y. G. Zhang and J. Wu, Ceram. Int., 2017, 43, 15747-15754.

33 L. H. H. O. Damink, P. J. Dijkstra, M. J. A. vanLuyn, P. B. vanWachem, P. Nieuwenhuis and J. Feijen, Biomaterials, 1996, 17, 765-773.

34 W. L. Yu, T. W. Sun, C. Qi, Z. Y. Ding, H. K. Zhao, F. Chen, D. Y. Chen, Y. J. Zhu, Z. M. Shi and Y. H. He, ACS Appl. Mater. Interfaces, 2017, 9, 3306-3317. 
35 N. Davidenko, C. F. Schuster, D. V. Bax, N. Raynal, R. W. Farndale, S. M. Best and R. E. Cameron, Acta Biomater., 2015, 25, 131-142.

36 G. S. Krishnakumar, N. Gostynska, E. Campodoni, M. Dapporto, M. Montesi, S. Panseri, A. Tampieri, E. Kon, M. Marcacci, S. Sprio and M. Sandri, Mater. Sci. Eng., C, 2017, 77, 594-605.

37 P. Kithva, L. Grondahl, D. Martin and M. Trau, J. Mater. Chem., 2010, 20, 381-389.

38 D. W. Hutmacher, Biomaterials, 2000, 21, 2529-2543.

39 V. Karageorgiou and D. Kaplan, Biomaterials, 2005, 26, 54745491.

40 S. H. Teng, E. J. Lee, P. Wang, D. S. Shin and H. E. Kim, J. Biomed. Mater. Res., Part B, 2008, 87B, 132-138.

41 X. Liu, L. A. Smith, J. Hu and P. X. Ma, Biomaterials, 2009, 30, 2252-2258.

42 Y. Wang, N. Van Manh, H. R. Wang, X. Zhong, X. Zhang and C. Y. Li, Int. J. Nanomed., 2016, 11, 2053-2067.

43 W. L. Yu, T. W. Sun, C. Qi, Z. Y. Ding, H. K. Zhao, S. C. Zhao, Z. M. Shi, Y. J. Zhu, D. Y. Chen and Y. H. He, Int. J. Nanomed., 2017, 12, 2293-2306.
44 K. I. Lee, V. F. Humphrey, T. G. Leighton and S. W. Yoon, Ultrasonics, 2007, 46, 323-330.

45 A. J. Engler, S. Sen, H. L. Sweeney and D. E. Discher, Cell, 2006, 126, 677-689.

46 C. M. Murphy, A. Matsiko, M. G. Haugh, J. P. Gleeson and F. J. O'Brien, J. Mech. Behav. Biomed. Mater., 2012, 11, 53-62.

47 S. J. Hollister, Nat. Mater., 2005, 4, 518-524.

48 S. B. Lee, J. S. Kwon, Y. K. Lee, K. M. Kim and K. N. Kim, J. Biomed. Mater. Res., Part B, 2012, 100B, 1935-1942.

49 X. M. Cheng, Y. B. Li, Y. Zuo, L. Zhang, J. D. Li and H. A. Wang, Mater. Sci. Eng., C, 2009, 29, 29-35.

50 E. Quinlan, S. Partap, M. M. Azevedo, G. Jell, M. M. Stevens and F. J. O'Brien, Biomaterials, 2015, 52, 358-366.

51 R. K. Singh, G. Z. Jin, C. Mahapatra, K. D. Patel, W. Chrzanowski and H. W. Kim, ACS Appl. Mater. Interfaces, 2015, 7, 8088-8098.

52 Y. F. Zhang, C. T. Wu, T. Friis and Y. Xiao, Biomaterials, 2010, 31, 2848-2856. 